\title{
Near surface stress analysis strategies for axisymmetric fretting
}

\author{
M RAMESH*, SATISH V KAILAS and K R Y SIMHA \\ Department of Mechanical Engineering, Indian Institute of Science, \\ Bangalore 560012 \\ e-mail: mramesh@mecheng.iisc.ernet.in
}

\begin{abstract}
Fretting is essentially a surface phenomenon, but bulk stresses and material properties contribute to subsequent failure. This feature of fretting demands a thorough understanding of near surface stresses under the joint action of normal, shear and thermal loading. Axisymmetric fretting is of great concern in piping and coupling design. In this paper, we develop design tools for Near Surface Analysis (NSA) for understanding axisymmetric fretting. Axisymmetric Fretting Analysis (AFA) becomes formidable owing to localised tractions that call for Fourier transform techniques. We develop two different NSA strategies based on two-dimensional plane strain models: 2D strip model (2DS) and half-plane Flamant model (2DF). We compare the results of 2DS and 2DF with the exact results for AFA obtained using Love's stress function in conjunction with Fourier transform. There is a good correspondence between stress components obtained from 2D-models.
\end{abstract}

Keywords. Solid cylinder; axisymmetric analysis; Fourier transform; 2D-models.

\section{Introduction}

Fretting is essentially a surface phenomenon although bulk stresses and materials properties contribute to subsequent failure. Stress analysis of relevance to fretting is mainly focused on crack initiation and propagation leading to gross failure of engineering components. In particular, initiation of microcracks in the vicinity of fretting depends on local stress and material properties such as yield stress, fracture toughness, etc. A recent overview on fretting fatigue highlights the key problems in aerospace applications (Farris et al 2003).

A common example of axisymmetric fretting is piping and shrink fit joints subjected to vibration (e.g. pressure vessels, piping, hub/shaft interface in turbine, etc.). Stress analysis of such joints need axisymmetric formulation. The axisymmetric analysis of cylinders subjected to localised loading on the lateral surface has been of interest for about past 75 years (Williams \& Ranson 2003). The problem of determining the state of stress in shrink fit components is widely reported in the literature (Yau \& Cakmak 1966; Conway \& Farnham 1967; Steven

${ }^{*}$ For correspondence 
1975; Spillers 1964; Lee 1995). Owing to the mathematical complexity of mixed boundary problems prescribing traction directly offers a valuable alternative. This alternative approach is not well explored and exploited in the literature.

An infinite cylinder subjected to constant pressure over a semi-infinite range was analysed by Tranter \& Craggs (1945). The problems of finite cylinder subjected to a pressure and shear tractions are solved in Timoshenko \& Goodier (1970) using Fourier series. Rankin \& Schenectady (1944) discussed the problem of infinitely long cylinder subjected to a band of pressure using Fourier transforms. Williams (1996) and Singh (1994) have given a brief history of allied problems reported in the literature. These analyses address the entire stress field in the cylinder without any emphasis on the near surface state of stress.

Dobromirsky \& Smith (1986) solved the problem of cylinder subjected to both pressure and shear bands, making the problem relevant to fretting. They included some results for state of stress at the surface and the near surface region of the cylinder. Axisymmetric analysis of fretting is generally found to be limited. However, the two-dimensional plane strain geometries of both strip and semi-infinite half-plane (Dini \& Nowell 2004; Hills et al 1993; Mugadu et al 2003; Szolwinski \& Farris 1996; Lee \& Mall 2004) are widely explored to understand fretting. Fellows et al (1995) studied the comparison of stresses in the strip and half-plane geometries in the context of fretting. Since fretting mainly depends on near surface stresses the effect of actual geometry of the component becomes less important. Thus, it is possible to take advantage of this feature to develop two-dimensional methods to understand the state of stress in axisymmetric fretting, provided such simplification is validated.

Considering the stresses in the near surface region only, the corresponding stresses obtained for the case of a two-dimensional strip could provide an approximate solution for an analogous axisymmetric problem. The axisymmetric analysis being the exact one, the two-dimensional strip analysis can be considered to be an approximate method as far as the stress in the near surface region is concerned. The objective of this paper is to develop design tools for Near Surface Analysis (NSA) for understanding axisymmetric fretting.

Following geometries are investigated for subsequent comparison of results:

(i) AFA infinitely long cylinder of radius R (figure 1).

(ii) 2DS infinitely long strip of half-width Y (2D plane strain case figure 2).

(iii) $2 \mathrm{DF}$ semi infinite half space (2D plane strain case figure 3 ).

Love's axisymmetric stress function and Airy's stress function are used in conjunction with the Fourier transform for the analysis of cylinder (AFA) and strip (2DS), respectively. For the

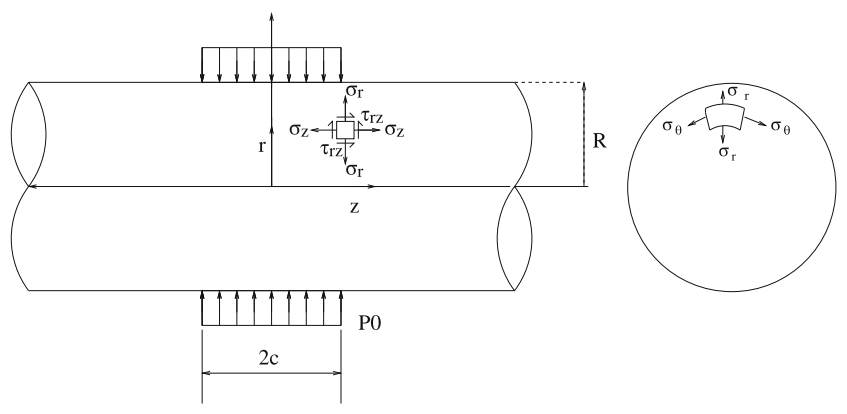

Figure 1. Homogeneous cylinder with cylindrical coordinate system. 


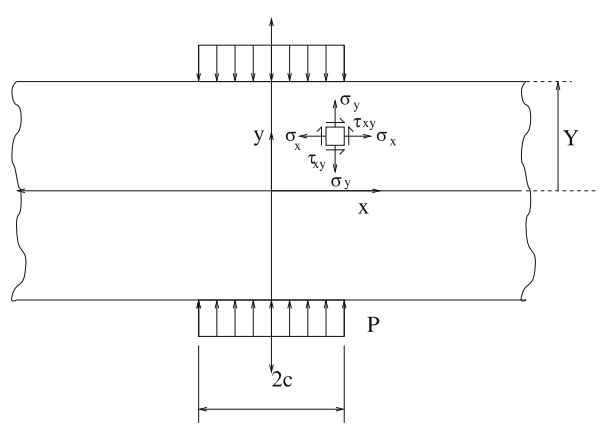

Figure 2. Strip with Cartesian coordinate system.

half-plane case, the Flamant solution is used as the Green's function for obtaining the stress, through integration.

In order to compare the near surface stresses from cylinder, strip and half-plane models, the applied traction profile is assumed to be known. Different profiles of relevance to fretting are considered including the classical Hertz solution for elastic contact. In the context of axisymmetric problems (AFA), bands of normal and shear tractions applied on the boundary of an infinitely long cylinder is a convenient starting point. Assuming the cylinder to be infinitely long permits the application of Fourier transform instead of Fourier series approximations valid for cylinder of finite length. Other than the Hertzian profile uniform and triangular bands of pressure and shear tractions (figure 4) are also analysed to provide better understanding of the problem. Further, they can be used to obtain approximate solution for mixed boundary problems (Bentall \& Johnson 1967; Nowell \& Hills 1988). The pressure profiles are centered around zero and the shear profiles are centered around $\pm c_{1}$, as shown in figure 4 . Thus $2 c_{1}$ is the separation between the two zones of loading of opposing shears each of width $2 c$. The material is assumed to be linear elastic, homogeneous and isotropic with Poisson's ratio $v=0 \cdot 25$. The loading is considered to be static.

\section{Formulation}

\subsection{AFA infinitely long cylinder using Fourier transform}

Axisymmetric formulation is based on Love's stress function $\Phi$ which satisfies the biharmonic equation (Timoshenko \& Goodier 1970). The stresses and displacements in cylindrical

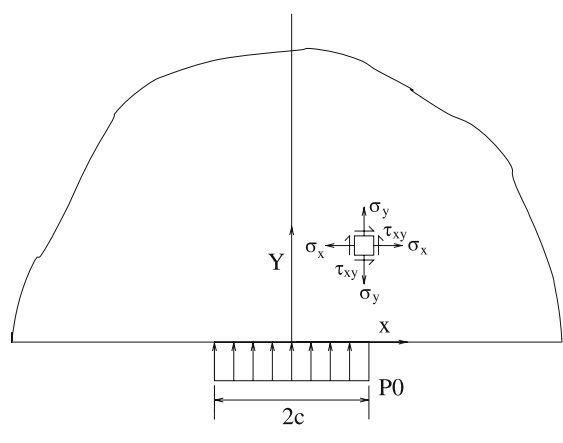

Figure 3. Semi infinite half-plane with Cartesian coordinate system. 

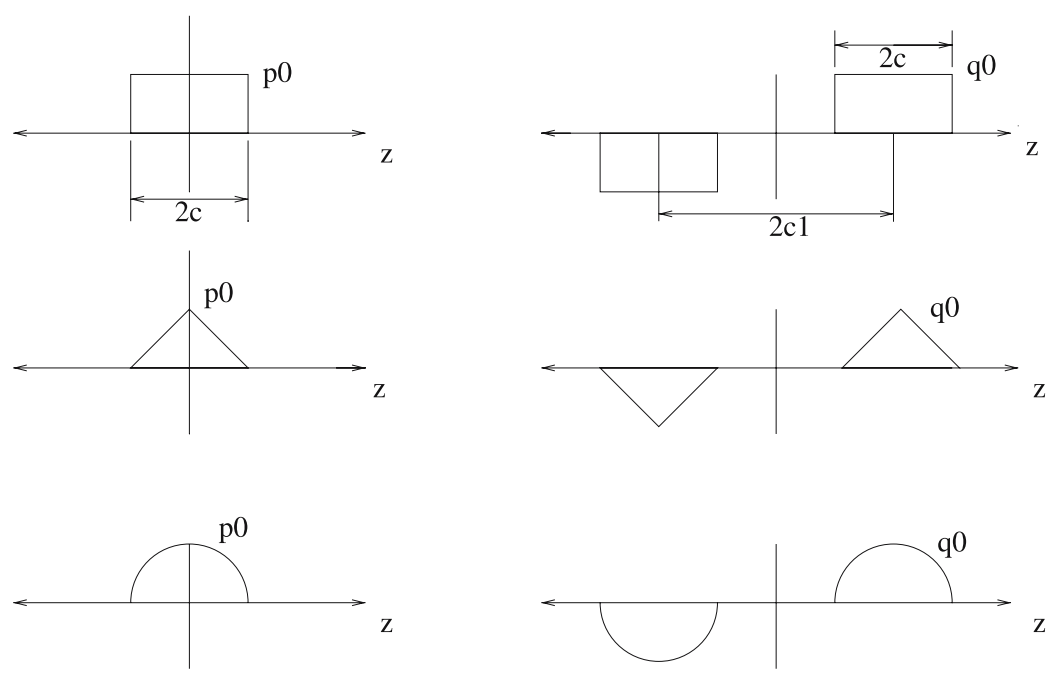

(a) Pressure

(b) Shear

Figure 4. Three different pressure and shear traction profiles.

coordinates are

$$
\begin{aligned}
2 G u_{r} & =-\frac{\partial^{2} \Phi}{\partial r \partial z} \\
2 G u_{z} & =2(1-v) \nabla^{2} \Phi-\frac{\partial^{2} \Phi}{\partial z^{2}} \\
\sigma_{r} & =\frac{\partial}{\partial z}\left[v \nabla^{2} \Phi-\frac{\partial^{2} \Phi}{\partial r^{2}}\right] \\
\sigma_{\theta} & =\frac{\partial}{\partial z}\left[v \nabla^{2} \Phi-\frac{1}{r} \frac{\partial \Phi}{\partial r}\right] \\
\sigma_{z} & =\frac{\partial}{\partial z}\left[(2-v) \nabla^{2} \Phi-\frac{\partial^{2} \Phi}{\partial z^{2}}\right] \\
\tau_{r z} & =\frac{\partial}{\partial r}\left[(1-v) \nabla^{2} \Phi-\frac{\partial^{2} \Phi}{\partial z^{2}}\right]
\end{aligned}
$$

where,

$$
\nabla^{2}=\frac{\partial^{2}}{\partial r^{2}}+\frac{1}{r} \frac{\partial}{\partial r}+\frac{\partial^{2}}{\partial z^{2}} .
$$

The strain displacement relations and the equilibrium equations are:

$$
\begin{aligned}
\epsilon_{r} & =\frac{\partial u_{r}}{\partial r} \\
\epsilon_{\theta} & =\frac{u}{r}
\end{aligned}
$$




$$
\begin{aligned}
& \epsilon_{z}=\frac{\partial u_{z}}{\partial z} \\
& \gamma_{r z}=\frac{\partial u_{r}}{\partial z}+\frac{\partial u_{z}}{\partial r} \\
& \frac{\partial \sigma_{r}}{\partial r}+\frac{\partial \tau_{r z}}{\partial z}+\frac{\sigma_{r}-\sigma_{\theta}}{r}=0 \\
& \frac{\partial \tau_{r z}}{\partial r}+\frac{\partial \sigma_{z}}{\partial z}+\frac{\tau_{r z}}{r}=0 .
\end{aligned}
$$

Whenever the stresses and displacements decay exponentially with increasing axial distance the method of Fourier sine or cosine integral transform can be employed. Since $\sigma_{r}, \sigma_{\theta}, \sigma_{z}$ and $u_{r}$ are symmetric and $\tau_{r z}, u_{z}$ are anti symmetric about $z=0, \Phi$ is anti symmetric. Fourier sine transform is taken for anti-symmetric and Fourier cosine transform is taken for symmetric components. Fourier sine transform appropriate for $\Phi$ is

$$
\begin{aligned}
& \bar{\Phi}(r, \xi)=\int_{0}^{\infty} \Phi(r, z) \sin (\xi z) d z \\
& \Phi(r, z)=\frac{2}{\pi} \int_{0}^{\infty} \bar{\Phi}(r, \xi) \sin (\xi z) d \xi .
\end{aligned}
$$

The corresponding stress and displacements in the Fourier space are:

$$
\begin{aligned}
2 G \bar{u}_{r} & =-\xi \frac{d \bar{\Phi}}{d r} \\
2 G \bar{u}_{z} & =2(1-v) \frac{d^{2} \bar{\Phi}}{d r^{2}}+2(1-v) \frac{1}{r} \frac{d \bar{\Phi}}{d r}-(1-2 v) \xi^{2} \bar{\Phi} \\
\bar{\sigma}_{r} & =-\xi\left[(1-v) \frac{d^{2} \bar{\Phi}}{d r^{2}}-\frac{v}{r} \frac{d \bar{\Phi}}{d r}+v \xi^{2} \bar{\Phi}\right] \\
\bar{\sigma}_{\theta} & =\xi\left[\nu \frac{d^{2} \bar{\Phi}}{d r^{2}}-\frac{1-v}{r} \frac{d \bar{\Phi}}{d r}-v \xi^{2} \bar{\Phi}\right] \\
\bar{\sigma}_{z} & =\xi\left[(2-v) \frac{d^{2} \bar{\Phi}}{d r^{2}}+\frac{2-v}{r} \frac{d \bar{\Phi}}{d r}-(1-v) \xi^{2} \bar{\Phi}\right] \\
\bar{\tau}_{r z} & =\frac{d}{d r}\left[(1-v) \frac{d^{2} \bar{\Phi}}{d r^{2}}+\frac{1-v}{r} \frac{d \bar{\Phi}}{d r}+v \xi^{2} \bar{\Phi}\right] .
\end{aligned}
$$

In the Fourier space the biharmonic equation $\left(\nabla^{2} \nabla^{2} \Phi=0\right)$ becomes

$$
\left(\frac{d^{2}}{d r^{2}}+\frac{1}{r} \frac{d}{d r}-\xi^{2}\right)\left(\frac{d^{2} \bar{\Phi}}{d r^{2}}+\frac{1}{r} \frac{d \bar{\Phi}}{d r}-\xi^{2} \bar{\Phi}\right)=0
$$


whose general solution is

$$
\bar{\Phi}=\frac{1}{\xi^{3}}\left[A_{1} I_{0}(\xi r)+A_{2} K_{0}(\xi r)+A_{3} \xi r I_{1}(\xi r)+A_{4} \xi r K_{1}(\xi r)\right],
$$

where $A_{j}(j=1,2,3$ and 4$)$ are functions of $\xi$ to be determined from applied boundary conditions. For the case of a solid cylinder as considered in this paper $A_{2}=A_{4}=0$. The functions $I_{0}, I_{1}, K_{0}$ and $K_{1}$ are the modified Bessel functions.

Using equation 18 for $\bar{\Phi}$, general solution for stress in the cylinder are (Rankin \& Schenectady 1944):

$$
\begin{aligned}
2 G u_{r} & =\int_{0}^{\infty} \frac{\xi r I_{0}(\xi r)-\rho I_{1}(\xi r)}{\xi \gamma} \frac{2 \bar{P}}{\pi} \cos (\xi z) d \xi \\
2 G u_{z} & =\int_{0}^{\infty} \frac{(\rho+4 v-4) I_{0}(\xi r)-\xi r I_{1}(\xi r)}{\xi \gamma} \frac{2 \bar{P}}{\pi} \sin (\xi z) d \xi \\
\sigma_{r} & =\int_{0}^{\infty} \frac{(1-2 v-\rho) I_{0}(\xi r)+\left(\xi r+\frac{\rho}{\xi r}\right) I_{1}(\xi r)}{\gamma} \frac{2 \bar{P}}{\pi} \cos (\xi z) d \xi \\
\sigma_{\theta} & =\int_{0}^{\infty} \frac{(1-2 v) I_{0}(\xi r)-\frac{\rho}{\xi r} I_{1}(\xi r)}{\gamma} \frac{2 \bar{P}}{\pi} \cos (\xi z) d \xi \\
\sigma_{z} & =\int_{0}^{\infty} \frac{(\rho+2 v-4) I_{0}(\xi r)-\xi r I_{1}(\xi r)}{\gamma} \frac{2 \bar{P}}{\pi} \cos (\xi z) d \xi \\
\tau_{r z} & =\int_{0}^{\infty} \frac{(\rho+2 v-2) I_{1}(\xi r)-\xi r I_{0}(\xi r)}{\gamma} \frac{2 \bar{P}}{\pi} \sin (\xi z) d \xi,
\end{aligned}
$$

where

$$
\begin{aligned}
\rho(\xi) & =-\frac{A_{1}(\xi)}{A_{3}(\xi)} \\
\gamma(\xi) & =-\frac{\bar{P}}{A_{3}(\xi)} \\
\bar{P} & = \begin{cases}\bar{\sigma}_{r}(R, \xi) & \text { for pressure boundary problem } \\
\bar{\tau}_{r z}(R, \xi) & \text { for shear boundary problem. }\end{cases}
\end{aligned}
$$

The expressions for $\rho$ and $\gamma$ (given in $\S 2 \cdot 1 \mathrm{a}$ and $2 \cdot 1 \mathrm{~b}$ ) depend on whether the problem is pressure prescribed or shear prescribed.

Owing to axisymmetric nature, the tangential stress $\sigma_{\theta}$ becomes a principal stress. The other two principal stresses are obtained from the following two expressions.

$$
\frac{\sigma_{r}+\sigma_{z}}{2} \pm\left[\left(\frac{\sigma_{r}-\sigma_{z}}{2}\right)^{2}+\tau_{r z}^{2}\right]^{\frac{1}{2}}
$$


Sorting these three values in descending order will give the maximum, intermediate and minimum principal stresses $\left(\sigma_{1}, \sigma_{2}, \sigma_{3}\right)$. The octahedral shear stress is then obtained from

$$
\tau_{\text {oct }}=\frac{1}{3}\left[\left(\sigma_{1}-\sigma_{2}\right)^{2}+\left(\sigma_{1}-\sigma_{3}\right)^{2}+\left(\sigma_{2}-\sigma_{3}\right)^{2}\right]^{\frac{1}{2}} .
$$

2.1a Pressure prescribed boundary $\sigma_{r}(R, \eta)=P(\eta)$ : The coordinate $\eta$ is common to all the three geometries considered, which is equivalent to $\mathrm{z}$ coordinate in cylinder problem and $\mathrm{x}$ coordinate in strip and semi-infinite half-plane problems. The expressions for $\rho$ and $\gamma$ are:

$$
\begin{aligned}
& \rho_{p}(\xi)=-\frac{A_{1}}{A_{3}}=2(1-v)+\xi R \frac{I_{0}(\xi R)}{I_{1}(\xi R)} \\
& \gamma_{p}(\xi)=-\frac{\bar{P}}{A_{3}}=(1-2 v-\rho) I_{0}(\xi R)+\left(\xi R+\frac{\rho}{\xi R}\right) I_{1}(\xi R) .
\end{aligned}
$$

2.1b Shear prescribed boundary $\tau_{r z}(R, \eta)=P(\eta)$ : The expressions for $\rho$ and $\gamma$ are:

$$
\begin{aligned}
& \rho_{s}(\xi)=-\frac{A_{1}}{A_{3}}=\frac{(1-2 \nu) I_{0}(\xi R)+\xi R I_{1}(\xi R)}{I_{0}(\xi R)-\frac{I_{1}(\xi R)}{\xi R}} \\
& \gamma_{s}(\xi)=-\frac{\bar{P}}{A_{3}}=[\rho-2(1-v)] I_{1}(\xi R)-\xi R I_{0}(\xi R) .
\end{aligned}
$$

\subsection{DS Infinitely long strip analysis using Fourier transform}

Plane strain formulation for two-dimensional strip is based on Airy's stress function $\Phi$ which satisfies the biharmonic equation (Timoshenko \& Goodier 1970). In Cartesian coordinates the components of stresses and displacements are:

$$
\begin{aligned}
2 G u_{y} & =(1-v) \int \frac{\partial^{2} \Phi}{\partial x^{2}} d y-v \frac{\partial \Phi}{\partial y} \\
2 G u_{x} & =(1-v) \int \frac{\partial^{2} \Phi}{\partial y^{2}} d x-v \frac{\partial \Phi}{\partial x} \\
\sigma_{y} & =\frac{\partial^{2} \Phi}{\partial x^{2}} \\
\sigma_{x} & =\frac{\partial^{2} \Phi}{\partial y^{2}} \\
\tau_{x y} & =-\frac{\partial^{2} \Phi}{\partial x \partial y} \\
\sigma_{w} & =v\left(\sigma_{x}+\sigma_{y}\right)=v \nabla^{2} \Phi,
\end{aligned}
$$

where

$$
\nabla^{2}=\frac{\partial^{2}}{\partial x^{2}}+\frac{\partial^{2}}{\partial y^{2}}
$$


The strain displacement relations and the equilibrium equations are:

$$
\begin{aligned}
& \epsilon_{y}=\frac{\partial u_{y}}{\partial y} \\
& \epsilon_{x}=\frac{\partial u_{x}}{\partial x} \\
& \epsilon_{z}=\frac{\partial u_{z}}{\partial z}=0 \\
& \gamma_{x y}=\frac{\partial u_{y}}{\partial x}+\frac{\partial u_{x}}{\partial y} \\
& \frac{\partial \sigma_{y}}{\partial y}+\frac{\partial \tau_{x y}}{\partial x}=0 \\
& \frac{\partial \tau_{x y}}{\partial y}+\frac{\partial \sigma_{x}}{\partial x}=0 .
\end{aligned}
$$

Since $\sigma_{y}, \sigma_{x}, \sigma_{w}$ and $u_{y}$ are symmetric and $\tau_{x y}, u_{x}$ are anti-symmetric about $x=0, \Phi$ is symmetric. Fourier cosine transform pair appropriate for $\Phi$ is:

$$
\begin{aligned}
& \bar{\Phi}(y, \xi)=\int_{0}^{\infty} \Phi(y, x) \cos (\xi x) d x \\
& \Phi(y, x)=\frac{2}{\pi} \int_{0}^{\infty} \bar{\Phi}(y, \xi) \cos (\xi x) d \xi
\end{aligned}
$$

The stresses and displacements in the Fourier space are:

$$
\begin{aligned}
2 G \bar{u}_{y} & =-(1-v) \xi^{2} \int \bar{\Phi} d y-v \frac{d \bar{\Phi}}{d y} \\
2 G \bar{u}_{x} & =\frac{1}{\xi}\left[(1-v) \frac{d^{2} \bar{\Phi}}{d y^{2}}+v \xi^{2} \bar{\Phi}\right] \\
\bar{\sigma}_{y} & =-\xi^{2} \bar{\Phi} \\
\bar{\tau}_{x y} & =\xi \frac{d \bar{\Phi}}{d y} \\
\bar{\sigma}_{x} & =\frac{d^{2} \bar{\Phi}}{d y^{2}} \\
\bar{\sigma}_{w} & =v\left[\frac{d^{2} \bar{\Phi}}{d y^{2}}-\xi^{2} \bar{\Phi}\right] .
\end{aligned}
$$


In the Fourier space the biharmonic equation $\left(\nabla^{2} \nabla^{2} \Phi=0\right)$ becomes

$$
\left(\frac{d^{2}}{d y^{2}}-\xi^{2}\right)\left(\frac{d^{2} \bar{\Phi}}{d y^{2}}-\xi^{2} \bar{\Phi}\right)=0
$$

whose general solution is

$$
\bar{\Phi}=\frac{1}{\xi^{2}}\left[A_{1} \cosh (\xi y)+A_{2} \sinh (\xi y)+A_{3} \xi \mathrm{y} \sinh (\xi y)+A_{4} \xi \mathrm{y} \cosh (\xi y)\right]
$$

where $A_{j}(j=1,2,3$ and 4$)$ are functions of $\xi$ to be determined from applied boundary conditions. Problem symmetry stipulates $A_{2}=A_{4}=0$.

Using equation 46 for $\bar{\Phi}$ a general solution for the stresses and displacements in the real space are (Sneddon 1951):

$$
\begin{aligned}
2 G u_{y} & =\int_{0}^{\infty} \frac{(-1+2 v-\rho) \sinh (\xi y)+\xi y \cosh (\xi y)}{\xi \gamma} \frac{2 \bar{P}}{\pi} \cos (\xi x) d \xi \\
2 G u_{x} & =\int_{0}^{\infty} \frac{(\rho+2 v-2) \cosh (\xi y)-\xi y \sinh (\xi y)}{\xi \gamma} \frac{2 \bar{P}}{\pi} \sin (\xi x) d \xi \\
\sigma_{y} & =\int_{0}^{\infty} \frac{-\rho \cosh (\xi y)+\xi y \sinh (\xi y)}{\gamma} \frac{2 \bar{P}}{\pi} \cos (\xi x) d \xi \\
\sigma_{x} & =\int_{0}^{\infty} \frac{(\rho-2) \cosh (\xi y)-\xi y \sinh (\xi y)}{\gamma} \frac{2 \bar{P}}{\pi} \cos (\xi x) d \xi \\
\sigma_{w} & =-\int_{0}^{\infty} \frac{2 v \cosh (\xi y)}{\gamma} \frac{2 \bar{P}}{\pi} \cos (\xi x) d \xi \\
\tau_{x y} & =\int_{0}^{\infty} \frac{(\rho-1) \sinh (\xi y)-\xi y \cosh (\xi y)}{\gamma} \frac{2 \bar{P}}{\pi} \sin (\xi x) d \xi,
\end{aligned}
$$

where

$$
\begin{gathered}
\rho(\xi)=-\frac{A_{1}(\xi)}{A_{3}(\xi)} \\
\gamma(\xi)=-\frac{\bar{P}}{A_{3}(\xi)} .
\end{gathered}
$$

The expressions for $\rho$ and $\gamma$ (given in $\S 2 \cdot 2 \mathrm{a}$ and $2 \cdot 2 \mathrm{~b}$ ) depend on whether the problem is pressure prescribed or shear prescribed one and $\bar{P}$ depend on the traction profile at the surface.

$$
\bar{P}= \begin{cases}\bar{\sigma}_{y}(Y, \xi) & \text { for pressure boundary problem } \\ \bar{\tau}_{x y}(Y, \xi) & \text { for shear boundary problem. }\end{cases}
$$


Owing to plane strain nature, the out of plane stress $\sigma_{w}$ is a principal stress. The other two principal stress are obtained from the following two expressions.

$$
\frac{\sigma_{y}+\sigma_{x}}{2} \pm\left[\left(\frac{\sigma_{y}-\sigma_{x}}{2}\right)^{2}+\tau_{x y}^{2}\right]^{\frac{1}{2}} .
$$

Sorting these three values in descending order will give the maximum, intermediate and minimum principal stresses $\left(\sigma_{1}, \sigma_{2}, \sigma_{3}\right)$. The octahedral stress is given by equation 24 .

2.2a Pressure prescribed boundary $\sigma_{y}(Y, \eta)=P(\eta)$ : Surface is shear free with a prescribed pressure profile i.e. $\tau_{x y}(Y, \eta)=0$ and $\sigma_{y}(Y, \eta)=P(\eta)$. The coordinate $\eta$ is common to all the three geometries considered, which is equivalent to $z$ coordinate in cylinder problem and $x$ coordinate in strip and semi-infinite half-plane problems. The expressions for $\rho$ and $\gamma$ are:

$$
\begin{aligned}
& \rho_{p}(\xi)=-\frac{A_{1}}{A_{3}}=1+\xi Y \frac{\cosh (\xi Y)}{\sinh (\xi Y)} \\
& \gamma_{p}(\xi)=-\frac{\bar{P}}{A_{3}}=-\rho \cosh (\xi Y)+\xi Y \sinh (\xi Y) .
\end{aligned}
$$

2.2b Shear prescribed boundary $\tau_{x y}(R, \eta)=P(\eta)$ : Expressions for $\rho$ and $\gamma$ are:

$$
\begin{aligned}
& \rho_{s}(\xi)=-\frac{A_{1}}{A_{3}}=\xi Y \frac{\sinh (\xi Y)}{\cosh (\xi Y)} \\
& \gamma_{s}(\xi)=-\frac{\bar{P}}{A_{3}}=(\rho-1) \sinh (\xi Y)-\xi Y \cosh (\xi Y) .
\end{aligned}
$$

\subsection{DF Semi infinite half-plane analysis using Flamant solution}

Through integration the stresses in the half-plane are obtained using Flamant solution as Green's function (Johnson 1985; Hills et al 1993).

For pressure boundary problem: $\sigma_{y}(0, \eta)=P(\eta)$

$$
\begin{aligned}
\sigma_{y} & =\frac{2 y^{3}}{\pi} \int_{-c}^{c} \frac{P(\eta) d \eta}{\left[(x-\eta)^{2}+y^{2}\right]^{2}} \\
\sigma_{x} & =\frac{2 y}{\pi} \int_{-c}^{c} \frac{P(\eta)(x-\eta)^{2} d \eta}{\left[(x-\eta)^{2}+y^{2}\right]^{2}} \\
\tau_{x y} & =\frac{2 y^{2}}{\pi} \int_{-c}^{c} \frac{P(\eta)(x-\eta) d \eta}{\left[(x-\eta)^{2}+y^{2}\right]^{2}} \\
\sigma_{w} & =v\left(\sigma_{x}+\sigma_{y}\right) .
\end{aligned}
$$

For shear boundary problem: $\tau_{x y}(0, \eta)=Q(\eta)$

$$
\begin{aligned}
\sigma_{y} & =\frac{2 y^{2}}{\pi} \int_{-c}^{c} \frac{Q(\eta)(x-\eta) d \eta}{\left[(x-\eta)^{2}+y^{2}\right]^{2}} \\
\sigma_{x} & =\frac{2}{\pi} \int_{-c}^{c} \frac{Q(\eta)(x-\eta)^{3} d \eta}{\left[(x-\eta)^{2}+y^{2}\right]^{2}}
\end{aligned}
$$




$$
\begin{aligned}
\tau_{x y} & =\frac{2 y}{\pi} \int_{-c}^{c} \frac{Q(\eta)(x-\eta)^{2} d \eta}{\left[(x-\eta)^{2}+y^{2}\right]^{2}} \\
\sigma_{w} & =v\left(\sigma_{x}+\sigma_{y}\right) .
\end{aligned}
$$

Results available in Hills et al (1993) and Johnson (1985) are reproduced here for completeness. Expressions for stress for the cases of uniform and triangular profiles are given here. For the case of Hertzian profile numerical integration is employed.

2.3a Uniform pressure profile $P(\eta)=p_{0}$ for $-c \leq \eta \leq c$ :

$$
\begin{aligned}
\sigma_{y} & =\frac{p_{0}}{\pi}\left[\tan ^{-1} \frac{c+x}{y}+\tan ^{-1} \frac{c-x}{y}+\frac{y(c+x)}{(c+x)^{2}+y^{2}}+\frac{y(c-x)}{(c-x)^{2}+y^{2}}\right] \\
\sigma_{x} & =\frac{p_{0}}{\pi}\left[\tan ^{-1} \frac{c+x}{y}+\tan ^{-1} \frac{c-x}{y}-\frac{y(c+x)}{(c+x)^{2}+y^{2}}-\frac{y(c-x)}{(c-x)^{2}+y^{2}}\right] \\
\tau_{x y} & =\frac{p_{0}}{\pi}\left[\frac{(c+x)^{2}}{(c+x)^{2}+y^{2}}-\frac{(c-x)^{2}}{(c-x)^{2}+y^{2}}\right] .
\end{aligned}
$$

2.3b Triangular pressure profile $P(\eta)=p_{0}\left(1-\frac{|\eta|}{c}\right)$ for $-c \leq \eta \leq c$ :

$$
\begin{aligned}
\sigma_{y}= & \frac{p_{0}}{\pi c}\left[(c+x) \tan ^{-1} \frac{c+x}{y}+(c-x) \tan ^{-1} \frac{c-x}{y}-2 x \tan ^{-1} \frac{x}{y}\right] \\
\sigma_{x}= & \frac{p_{0} y}{\pi c} \ln \frac{\left(x^{2}+y^{2}\right)^{2}}{\left[(c+x)^{2}+y^{2}\right]\left[(c-x)^{2}+y^{2}\right]} \\
& +\frac{p_{0}}{\pi c}\left[(c+x) \tan ^{-1} \frac{c+x}{y}+(c-x) \tan ^{-1} \frac{c-x}{y}-2 x \tan ^{-1} \frac{x}{y}\right] \\
\tau_{x y}= & -\frac{p_{0} y}{\pi c}\left[\tan ^{-1} \frac{c+x}{y}-\tan ^{-1} \frac{c-x}{y}-2 \tan ^{-1} \frac{x}{y}\right] .
\end{aligned}
$$

2.3c Uniform shear profile $Q(\eta)=q_{0}$ for $-c \leq \eta \leq c$ :

$$
\begin{aligned}
\sigma_{y} & =-\frac{q_{0}}{\pi}\left[\frac{y^{2}}{(c+x)^{2}+y^{2}}-\frac{y^{2}}{(c-x)^{2}+y^{2}}\right] \\
\sigma_{x} & =\frac{q_{0}}{\pi}\left[\ln \frac{(c+x)^{2}+y^{2}}{(c-x)^{2}+y^{2}}+\frac{y^{2}}{(c+x)^{2}+y^{2}}-\frac{y^{2}}{(c-x)^{2}+y^{2}}\right] \\
\tau_{x y} & =\frac{q_{0}}{\pi}\left[\tan ^{-1} \frac{c+x}{y}+\tan ^{-1} \frac{c-x}{y}-\frac{y(c+x)}{(c+x)^{2}+y^{2}}-\frac{y(c-x)}{(c-x)^{2}+y^{2}}\right] .
\end{aligned}
$$

2.3d Triangular shear profile $Q(\eta)=q_{0}\left(1-\frac{|\eta|}{c}\right)$ for $-c \leq \eta \leq c$ :

$$
\sigma_{y}=\frac{q_{0} y}{\pi c}\left[\tan ^{-1} \frac{c-x}{y}-\tan ^{-1} \frac{c+x}{y}+2 \tan ^{-1} \frac{x}{y}\right]
$$




$$
\begin{aligned}
\sigma_{x}= & \frac{q_{0}}{\pi c}\left[(c+x) \ln \frac{(c+x)^{2}+y^{2}}{x^{2}+y^{2}}-(c-x) \ln \frac{(c-x)^{2}+y^{2}}{x^{2}+y^{2}}\right] \\
& +\frac{q_{0}}{\pi c} 3 y\left[\tan ^{-1} \frac{c+x}{y}-\tan ^{-1} \frac{c-x}{y}-2 \tan ^{-1} \frac{x}{y}\right] \\
\tau_{x y}= & \frac{q_{0} y}{\pi c} \ln \frac{\left(x^{2}+y^{2}\right)^{2}}{\left[(c+x)^{2}+y^{2}\right]\left[(c-x)^{2}+y^{2}\right]} \\
& +\frac{q_{0}}{\pi c}\left[(c+x) \tan ^{-1} \frac{c+x}{y}+(c-x) \tan ^{-1} \frac{c-x}{y}-2 x \tan ^{-1} \frac{x}{y}\right] .
\end{aligned}
$$

Various loading cases shown in figure 4 yield different near surface stress fields. The Fourier transformed boundary conditions are:

Uniform pressure profile: $p(\eta)=p_{0}$ for $-c \leq \eta \leq c$

$$
\bar{P}(\xi)=\int_{0}^{\infty} P(\eta) \cos (\xi \eta) d \eta=p_{0} \frac{\sin (\xi c)}{\xi}
$$

Triangular pressure profile: $p(\eta)=p_{0}\left[1-\frac{|\eta|}{c}\right]$ for $-c \leq \eta \leq c$

$$
\bar{P}(\xi)=\int_{0}^{\infty} P(\eta) \cos (\xi \eta) d \eta=2 p_{0} \frac{\sin ^{2}(\xi c / 2)}{\xi^{2} c} .
$$

Hertzian pressure profile: $p(\eta)=p_{0} \sqrt{1-\frac{\eta^{2}}{c^{2}}}$ for $-c \leq \eta \leq c$

$$
\bar{P}(\xi)=\int_{0}^{\infty} P(\eta) \cos (\xi \eta) d \eta=p_{0} \frac{\pi}{2} \frac{J_{1}(\xi c)}{\xi},
$$

where $J_{1}$ is the Bessel function of first order.

Similar expressions are obtainable for uniform shear, triangular shear and Hertzian shear profiles.

\section{Results and discussion}

In order to generate numerical results, the radius $R$ of the cylinder and half-width $Y$ of the strip are taken as unity. Pressure or shear is applied along the lateral surface over a length of $c=0.15 R=0.15 Y$ with the peak pressure $p_{o}=-100 \mathrm{MPa}$ for all three profiles. For the case of shear boundary problem two similar profiles of opposite sign are applied for equilibrium. However, the interaction of stress due to each profile is avoided by taking a large center to center distance of 10 times the profile width (i.e. $c_{1}=10 c$ ). Stresses are obtained for all the cases for subsequent comparison. For numerical calculation the integrals are split into two parts since the direct calculation of the integrals becomes difficult for some cases. For example consider the tangential stress $\sigma_{\theta}(R, z)$.

$$
\sigma_{\theta}(R, z)=\frac{2}{\pi} \int_{0}^{\infty} \frac{(1-2 \nu) I_{0}(\xi R)-\frac{\rho}{\xi R} I_{1}(\xi R)}{\gamma} \bar{P} \cos (\xi z) d \xi
$$




$$
\begin{aligned}
\sigma_{\theta}(R, z)= & \frac{2}{\pi} \int_{0}^{\infty}\left[\frac{(1-2 v) I_{0}(\xi R)-\frac{\rho}{\xi R} I_{1}(\xi R)}{\gamma}-s\right] \bar{P} \cos (\xi z) d \xi \\
& +s \frac{2}{\pi} \int_{0}^{\infty} \bar{P} \cos (\xi z) d \xi \\
= & \frac{2}{\pi} \int_{0}^{\infty}\left[\frac{(1-2 v) I_{0}(\xi R)-\frac{\rho}{\xi R} I_{1}(\xi R)}{\gamma}-s\right] \bar{P} \cos (\xi z) d \xi \\
& +s P(z),
\end{aligned}
$$

where

$$
s=\lim _{\xi \rightarrow \infty} \frac{(1-2 \nu) I_{0}(\xi R)-\frac{\rho}{\xi R} I_{1}(\xi R)}{\gamma}
$$

and $\mathrm{P}(\mathrm{z})$ is the applied traction at the surface.

The limiting values of $s$ are found to be $1,0.5$ and 1 for $\sigma_{r}, \sigma_{\theta}$ and $\sigma_{z}$, respectively, for the case of pressure prescribed problem. The remaining integral can be easily calculated numerically. For numerical integration the range of $\xi$ is taken to be $\xi=10^{-5}$ to 700 with the interval being $0 \cdot 1$, using actual values of Bessel functions in MATLAB.

Since fretting is mainly controlled by the near surface stresses, results are given for $r=$ $0.9 R, 0.95 R$ and $0.99 R$. Similarly, results up to an axial distance of $3 \mathrm{c}$ from the middle are shown.

Exact stresses obtained from axisymmetric analysis of the cylinder are compared with the corresponding stresses in the strip and half-plane geometries. For comparison the following stresses are considered to be equivalent in all three geometries. (i) radial stress $\sigma_{r}$ and transverse normal stress $\sigma_{y}$, (ii) shear stress $\tau_{r z}$ and shear stress $\tau_{x y}$, (iii) axial stress $\sigma_{z}$ and longitudinal normal stress $\sigma_{x}$, (iv) tangential stress $\sigma_{\theta}$ and out of plane normal stress $\sigma_{w}$.

Though strictly speaking $\sigma_{\theta}$ in the cylinder is not equivalent to $\sigma_{w}$ in the strip problem, they are compared to examine if they have any corresponding results.

\subsection{Pressure prescribed boundary}

Figures 5, 6 and 7 show the stresses plotted with respect to the axial distance for the pressure boundary problem with three different pressure profiles. The applied pressure is well shown by $\sigma_{r}$ at $r=0.99 R$. In each plot the cylinder results are compared with strip and semi-infinite half-plane geometries. In all the figures solid line represents the stress in cylinder while dashed line and dash-dot line represent the stresses in the strip and half-plane, respectively.

Figure 5 corresponds to the case of uniform pressure band. The transverse normal stress $\sigma_{y}$ obtained from both the strip and half-plane models match well with $\sigma_{r}$ in the cylinder for the three radii considered. Also there is a good match between the results of shear stress $\tau_{x y}$ from strip and half-plane models to $\tau_{r z}$ in the cylinder. $\sigma_{x}$ in the strip has a good correspondence with $\sigma_{z}$ whereas $\sigma_{x}$ in the half-plane differs significantly. The out of plane stress $\sigma_{w}$ obtained from both the strip and half-plane has a close correspondence with each other but they do not match with the results of $\sigma_{\theta}$ obtained from the cylinder. It can be shown that the out of plane stress $\sigma_{w}$ from half-plane model match with $\sigma_{\theta}$ only in the case of radius tending to 

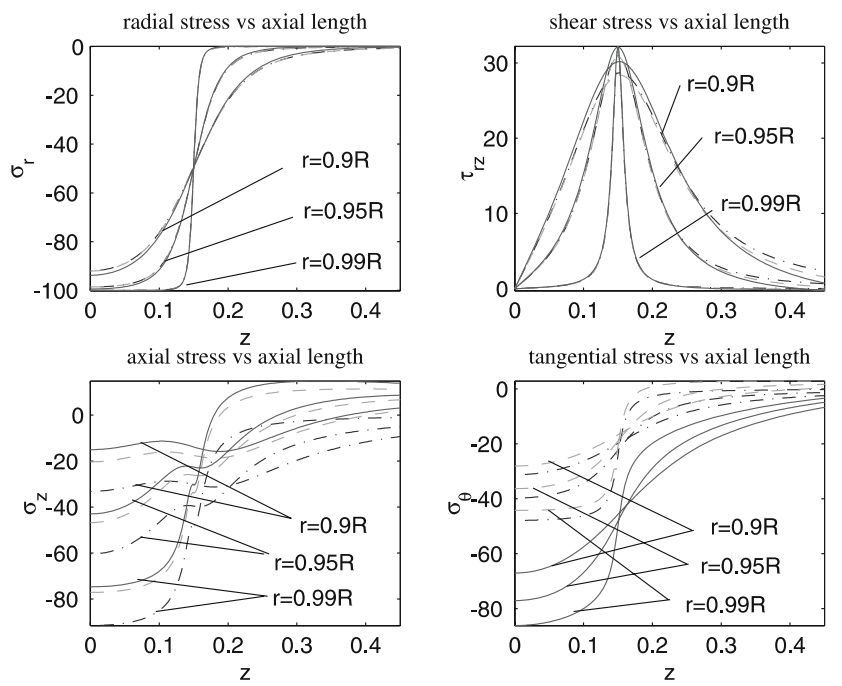

Figure 5. Stresses in the cylinder, strip and half-plane subjected to uniform band pressure.

infinity. The expressions for $\sigma_{\theta}$ and $\nu\left(\sigma_{r}+\sigma_{z}\right)$ obtained from equations 3,5 and 4 reveal this fact.

$$
\begin{aligned}
\sigma_{\theta} & =\frac{\partial}{\partial z}\left[v \nabla^{2} \Phi-\frac{1}{r} \frac{\partial \Phi}{\partial r}\right] \\
v\left(\sigma_{r}+\sigma_{z}\right) & =\frac{\partial}{\partial z}\left[v \nabla^{2} \Phi+\frac{v}{r} \frac{\partial \Phi}{\partial r}\right] .
\end{aligned}
$$
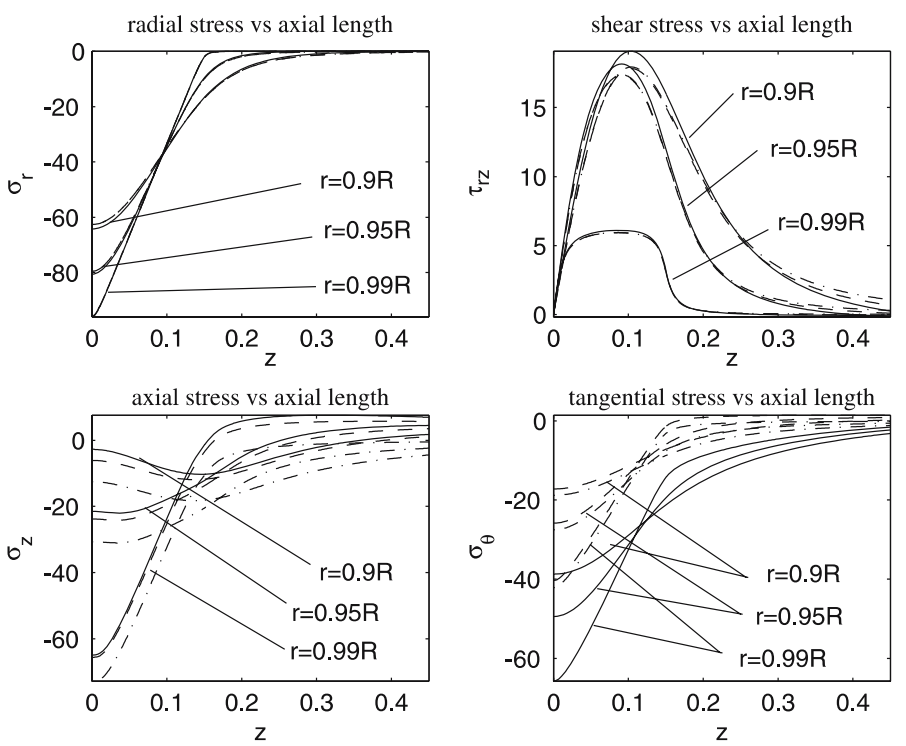

Figure 6. Stresses in the cylinder, strip and half-plane subjected to triangular band pressure. 

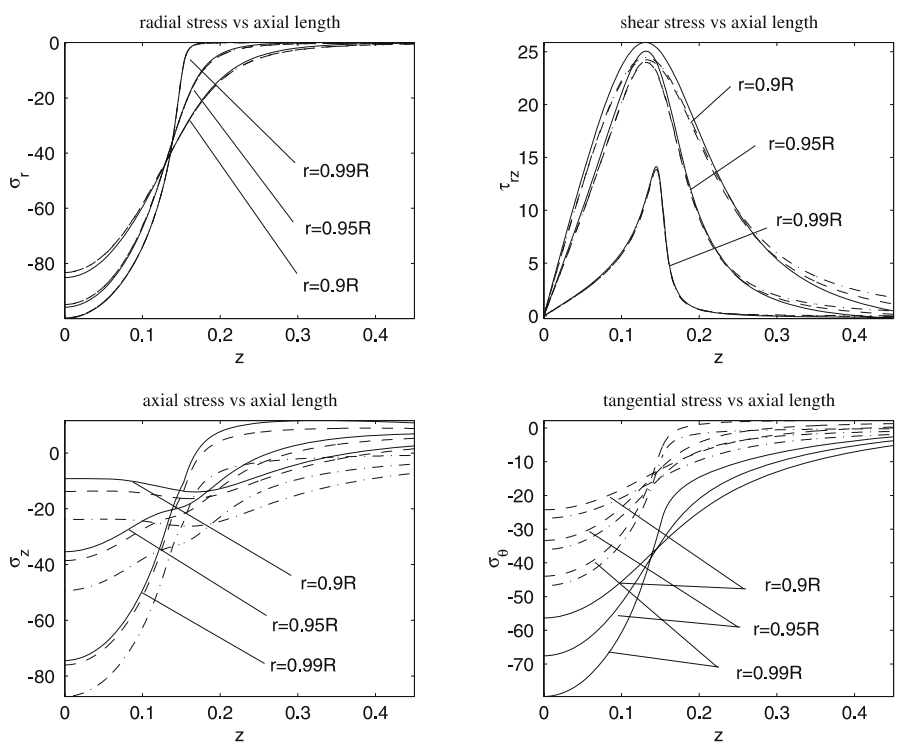

Figure 7. Stresses in the cylinder, strip and half-plane subjected to Hertzian band pressure.

Further, consider $\sigma_{\theta}(R, z)$ in the cylinder as given in equation 67. Substituting for $\bar{P}$ from equation 62 and multiplying the numerator and denominator by $R$ we get

$$
\begin{aligned}
\sigma_{\theta}(R, z)= & \frac{2}{\pi} \int_{0}^{\infty}\left[\frac{(1-2 v) I_{0}(\xi R)-\frac{\rho}{\xi R} I_{1}(\xi R)}{\gamma}-s\right] \frac{\sin (\xi c)}{\xi R} \cos (\xi z) d(\xi R) \\
& +s P(z) .
\end{aligned}
$$

Substituting $\xi R=\xi^{\prime}$ the equation becomes

$$
\begin{aligned}
\sigma_{\theta}(R, z)= & \frac{2}{\pi} \int_{0}^{\infty}\left[\frac{(1-2 v) I_{0}\left(\xi^{\prime}\right)-\frac{\rho}{\xi^{\prime}} I_{1}\left(\xi^{\prime}\right)}{\gamma}-s\right] \frac{\sin \left(\xi^{\prime} c / R\right)}{\xi^{\prime}} \cos \left(\xi^{\prime} z / R\right) d\left(\xi^{\prime}\right) \\
& +s P(z) .
\end{aligned}
$$

The expression for $s$ now becomes

$$
s=\lim _{\xi^{\prime} \rightarrow \infty} \frac{(1-2 \nu) I_{0}\left(\xi^{\prime}\right)-\frac{\rho}{\xi^{\prime}} I_{1}\left(\xi^{\prime}\right)}{\gamma}=\frac{1}{2} .
$$

In the limit $R$ tending to infinity, $\sin \left(\xi^{\prime} c / R\right)$ becomes zero for a given value of $c$ and the integral vanishes. Thus

$$
\lim _{R \rightarrow \infty} \sigma_{\theta}(R, z)=\frac{1}{2} P(z)=\frac{p_{0}}{2} H(c-z),
$$

where $H$ is the Heaviside step function. Similarly for the half-plane case from equations 56 and 58

$$
\sigma_{w}(y, x)=v\left(\sigma_{x}+\sigma_{y}\right)=2 v \frac{p_{0}}{\pi}\left[\tan ^{-1} \frac{c+x}{y}+\tan ^{-1} \frac{c-x}{y}\right]
$$


which at $y=0$ gives

$$
\sigma_{w}(0, x)=2 v \frac{p_{0}}{\pi} \pi H(c-x)=2 v p_{0} H(c-x)=\frac{p_{0}}{2} H(c-x) .
$$

From equations 73 and 75 it is shown that $\sigma_{\theta}$ in the cylinder approaches the out of plane stress $\sigma_{w}$ of half-plane model. Also it can be understood from above equations that increasing the radius for a given width of the pressure band is equivalent to decreasing the width of the pressure band for a given radius.

Similarly, for triangular and Hertzian pressure profiles, there is a good match between the three different geometries for $\sigma_{r}$ and $\tau_{r z} . \sigma_{x}$ in the strip predicts approximately the axial stress $\sigma_{z}$ in the cylinder whereas $\sigma_{x}$ in the half-plane differs from $\sigma_{z}$ by almost a constant value.

In general, for the case of pressure prescribed problem the strip model predicts $\sigma_{r}, \sigma_{z}$ and $\tau_{r z}$ in a cylinder approximately. The half-plane Flamant model predicts only $\sigma_{r}$ and $\tau_{r z}$ but not $\sigma_{z}$. However, both models fail to predict $\sigma_{\theta}$.

\subsection{Shear prescribed boundary}

For the case of shear boundary problem, figures 8,9 and 10 show the stresses plotted with respect to axial distance from the middle of the profile to an axial distance of three times the half profile width (i.e. $z=1.5$ to $z=1.5+3 c=1.95$ ). Similar to the pressure prescribed problem there is a close match between the stress in all the three geometries with regard to $\sigma_{r}$ and $\tau_{r z} . \sigma_{x}$ in the strip and half-plane differs from $\sigma_{z}$ by a uniform difference. The difference is smaller for the case of strip and is larger for the case of half-plane. In contrast to the pressure prescribed problem $\sigma_{w}$ in the strip tends to match with $\sigma_{z}$ as the surface is approached.

As far as fretting is considered, the axial stress $\sigma_{z}$ is an important component in propagating the crack initiated. In the pressure boundary problem, the axial stress $\sigma_{z}$ becomes tensile
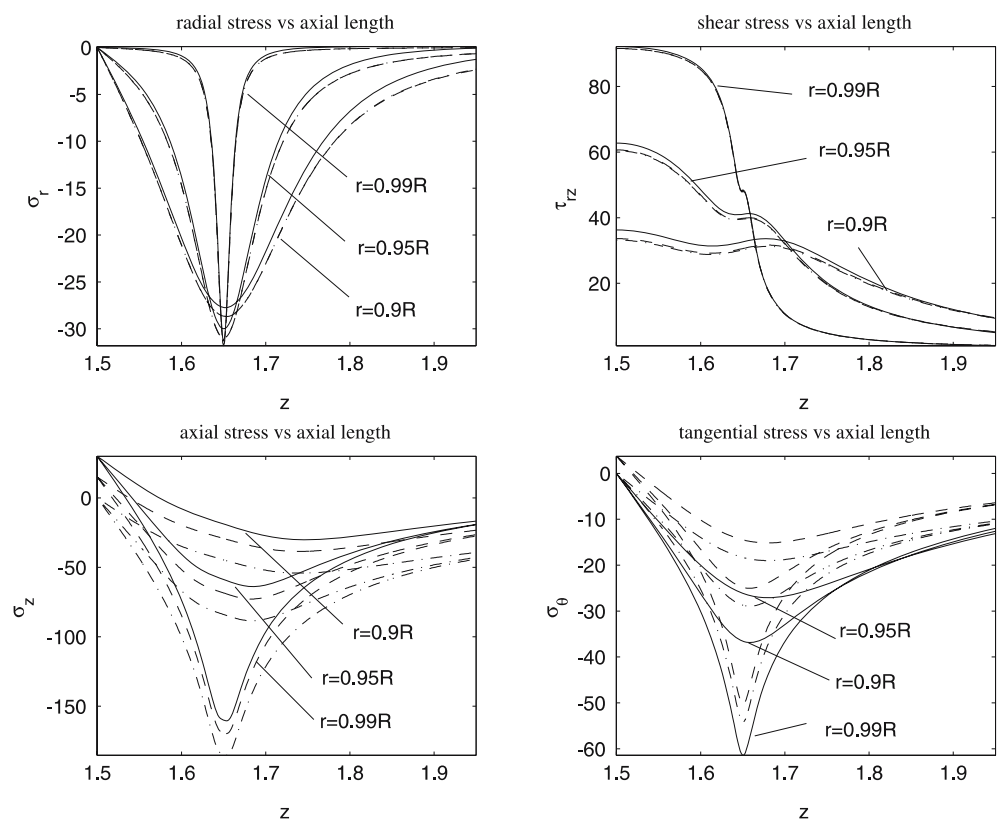

Figure 8. Stresses in the cylinder, strip and half-plane subjected to uniform shear band. 

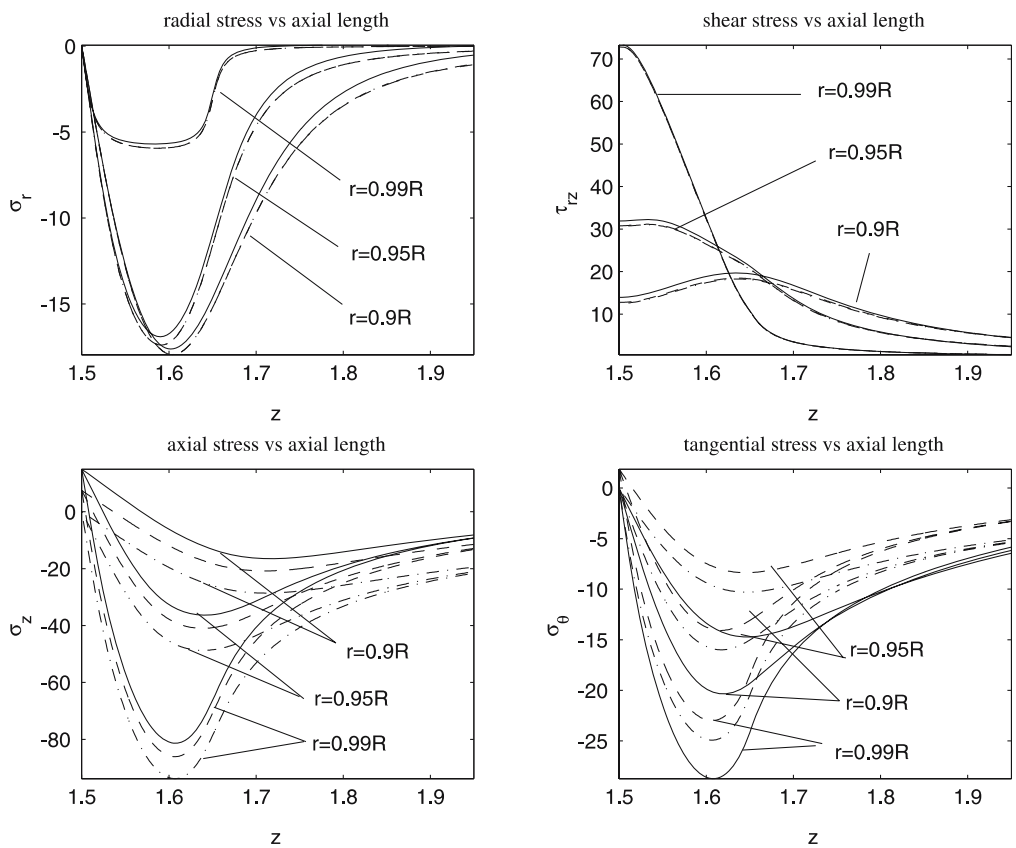

Figure 9. Stresses in the cylinder, strip and half-plane subjected to triangular shear band.
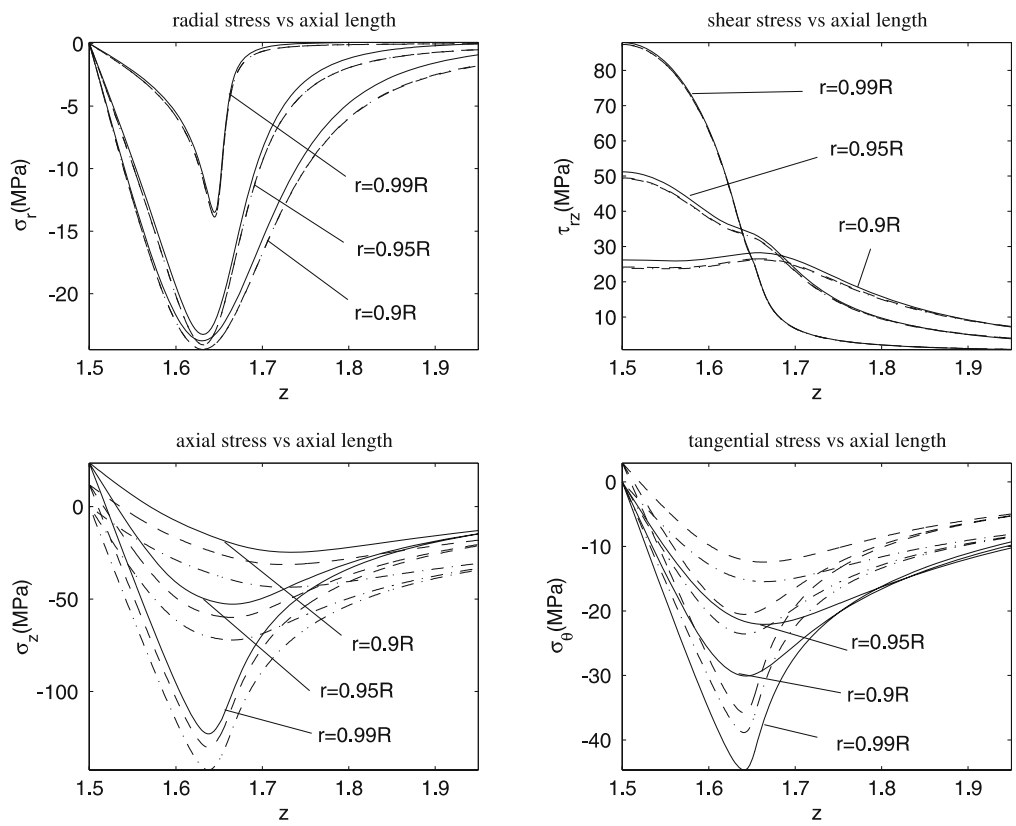

Figure 10. Stresses in the cylinder, strip and half-plane subjected to Hertzian shear band. 

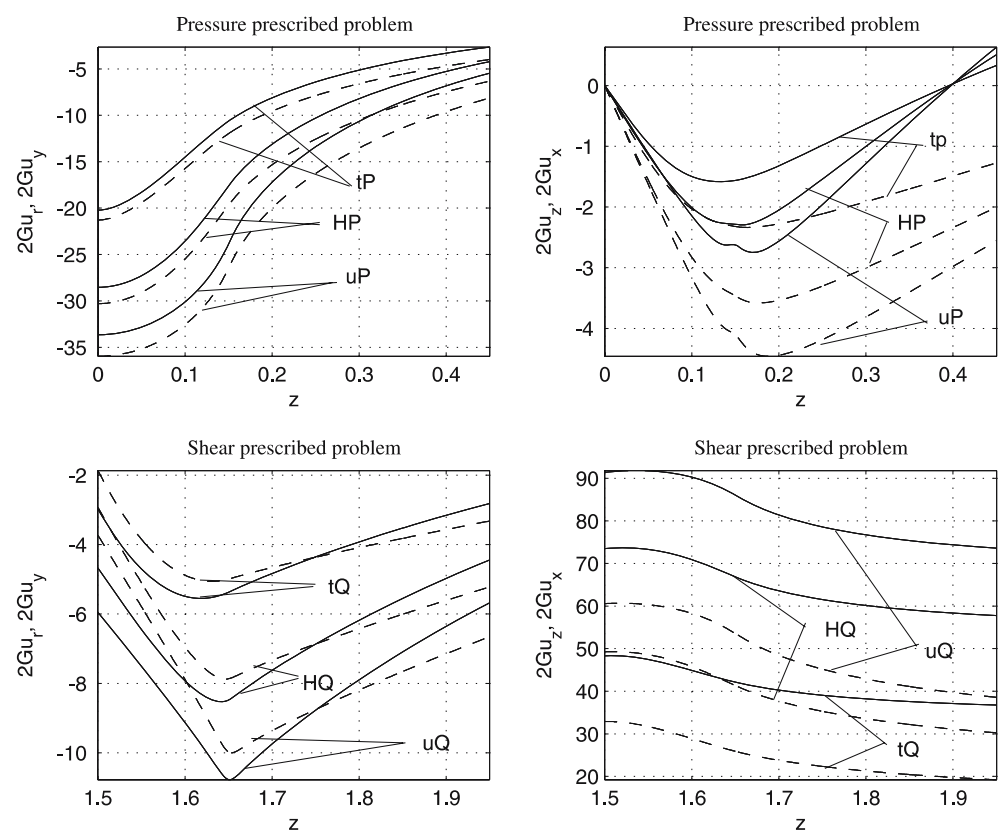

Figure 11. Near surface displacements at $r=0.99 R$ in the cylinder and strip (u-uniform; t-triangular; H-Hertzian; P-pressure; Q-shear).

at the edge of the profile width, however the magnitude is less compared to that of applied radial compressive pressure (figures 5, 6 and 7). For the case of shear boundary problem the magnitude of axial stress $\sigma_{z}$ is quite higher than the magnitude of shear stress $\tau_{r z}$ (figures 8 , 9 and 10). Even though the axial stress is shown to be compressive in the plot it is tensile in the left portion of profile $(z=1.5-3 c=1.05$ to $z=1.5)$ which is not shown in the plot. Also the compressive stress becomes tensile when the direction of applied shear traction is reversed.

\subsection{Near surface displacements}

Figure 11 shows the near surface displacements $(r=0.99 R)$ for the cases of cylinder and strip. The displacement magnitudes are higher for the cases of uniform pressure and shear profiles due to the fact that the total load is higher than the triangular and Hertzian profiles. The strip model is able to predict approximately the radial displacement but not the axial displacement.

\subsection{Octahedral shear stress}

Figure 12 shows the plots of octahedral shear stress $\tau_{\text {oct }}$ on the plane $z=0$ with radius $r=0.5$ to 0.99 , for the case of pressure prescribed boundary. The plots show that $2 \mathrm{D}$ models poorly predict $\tau_{\text {oct }}$ in the cylinder especially in the near surface region for all the three pressure profiles. The inherent inability of the $2 \mathrm{D}$ models to predict $\sigma_{\theta}$ for the pressure prescribed problem is reflected in predicting $\tau_{o c t}$. A better prediction of $\tau_{o c t}$ is expected for the shear prescribed problem because the prediction of all the stress components is better for the shear prescribed problem when compared to the pressure prescribed problem. 

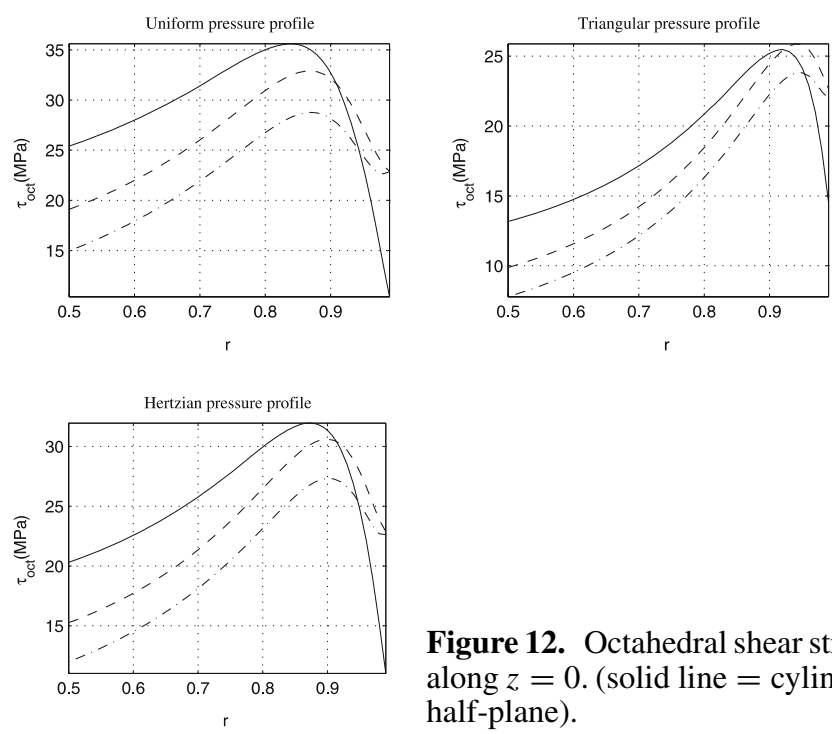

Figure 12. Octahedral shear stress in the cylinder, strip and half-plane along $z=0$. (solid line $=$ cylinder; dash line $=$ strip; dash-dot line $=$ half-plane).

The location of maximum octahedral shear stress is an important issue in contact phenomenon to predict failure by yielding. Deeper this location of maximum shear, higher the margin of safety is likely to be. Also, it can be observed that the position of the maximum octahedral shear stress $\tau_{o c t}$ in the cylinder lies deeper than that of strip and half-plane models. The contours of $\tau_{o c t}$ in the near surface region very close to the end of the pressure band is shown in figure 13 for the case of uniform pressure.

The line of steepest descent is indicated for the cylinder and strip models. This line of steepest descent is absent in the 2D Flamant model. There is some previous work on this
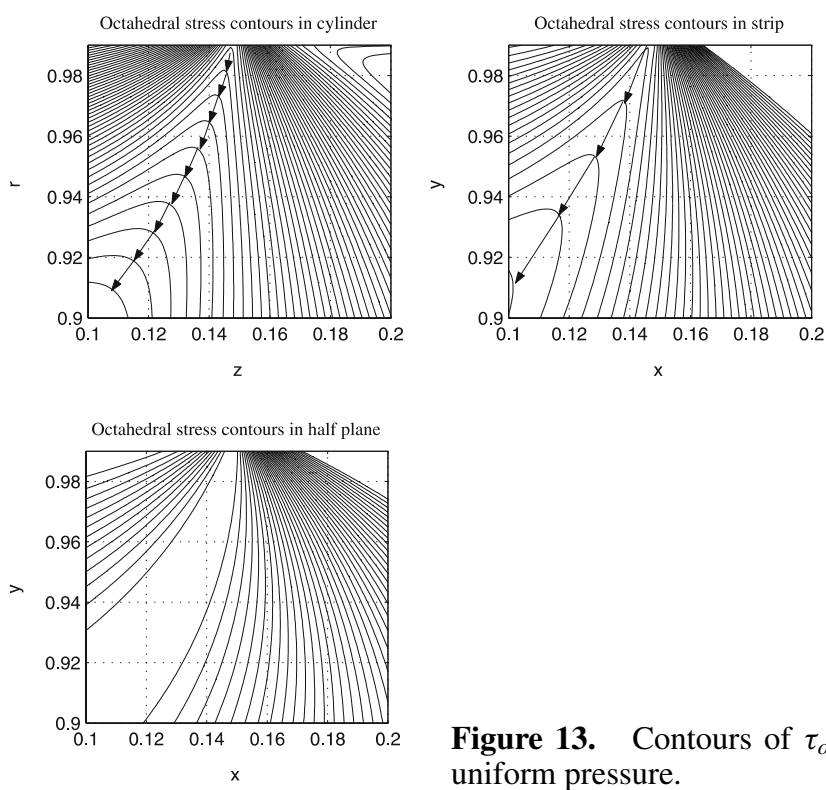

Figure 13. Contours of $\tau_{o c t}$ at the end of pressure band due to uniform pressure. 

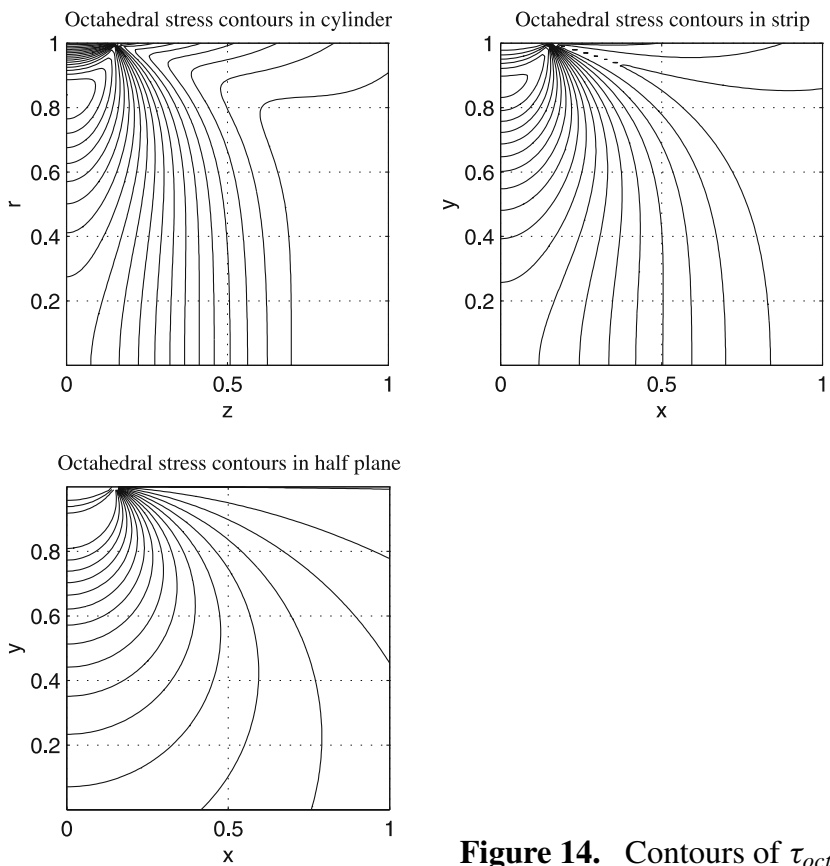

Figure 14. Contours of $\tau_{\text {oct }}$ due to uniform pressure.

path of steepest descent in the context of predicting the crack path in mixed mode fracture (Boniface \& Simha 1991). The contours of $\tau_{\text {oct }}$ over a region of $r=0$ to $R$ and $z=0$ to $R$ are given in figures 14,15 and 16.
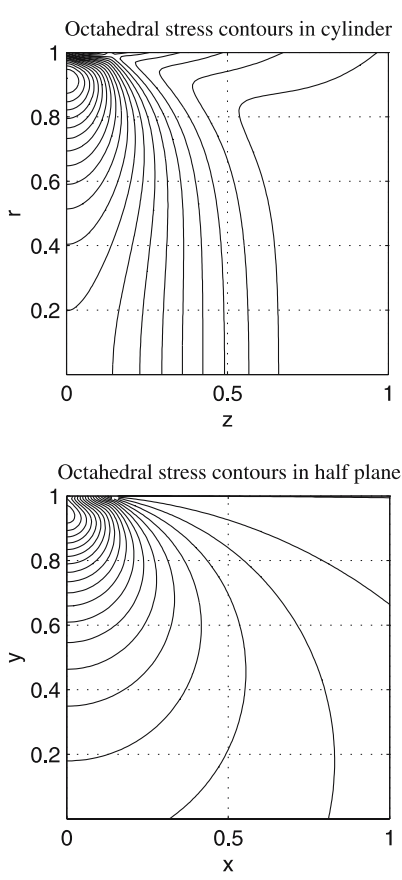

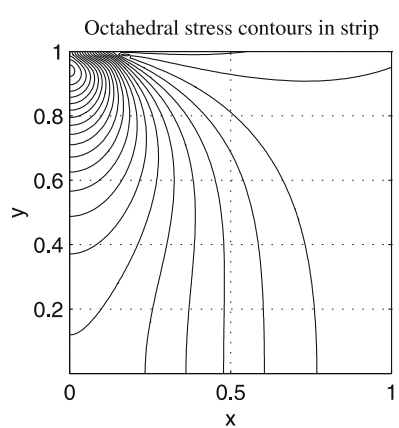

Figure 15. Contours of $\tau_{o c t}$ due to triangular pressure. 

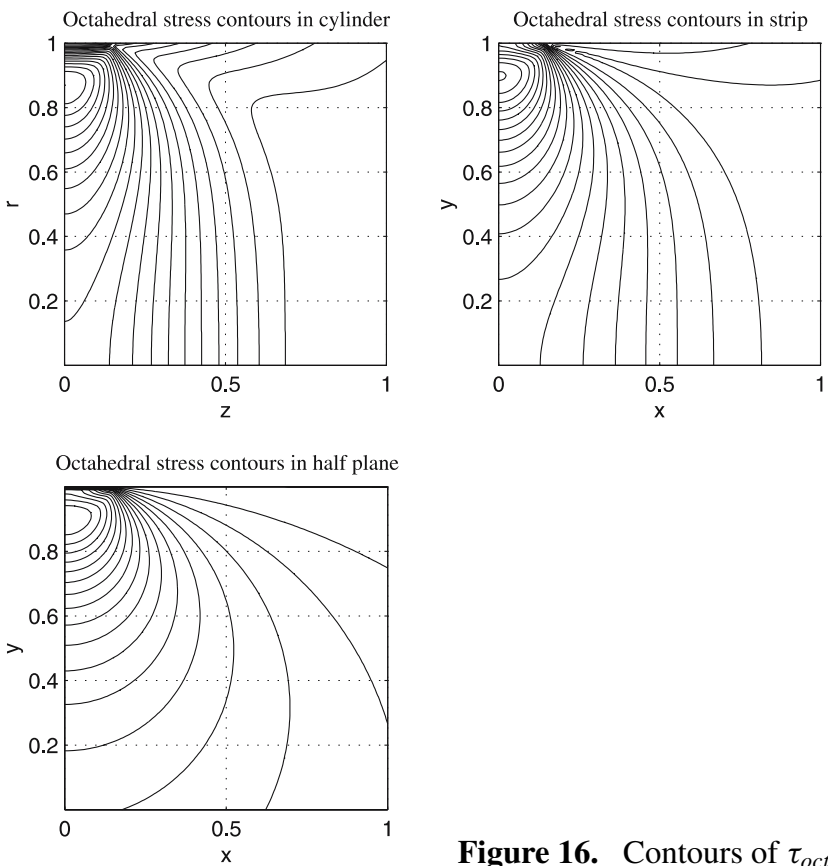

Figure 16. Contours of $\tau_{o c t}$ due to Hertzian pressure.

\subsection{Fretting loading}

The general fretting contact problem such as the shrink fit generates both pressure and shear tractions at the surface. Hence a full solution requires a superposition of results of both the pressure boundary and shear boundary problems (Dobromirsky \& Smith 1986).

(a)

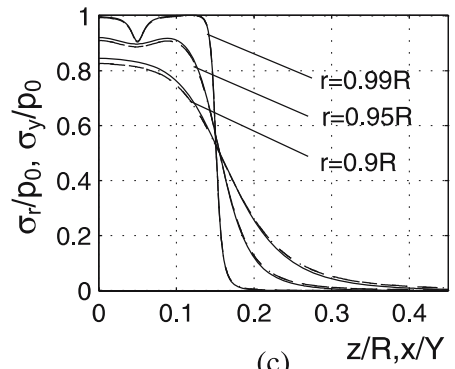

(c)

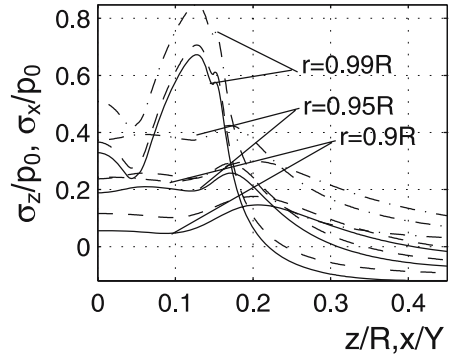

(b)

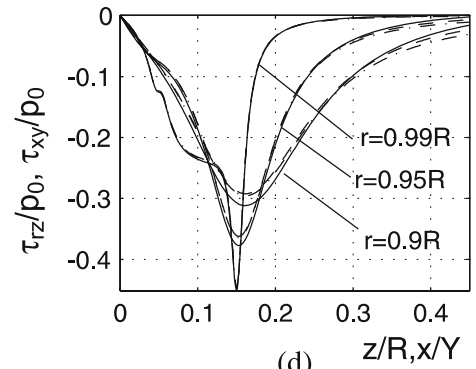

(d)

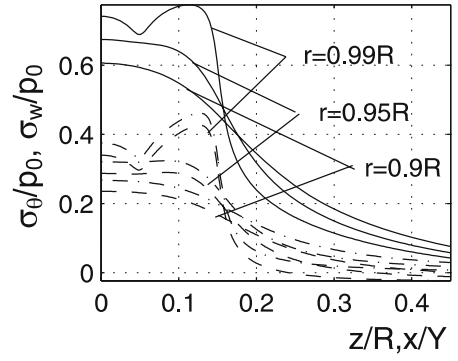

Figure 17. Stresses due to uniform pressure and shear. 


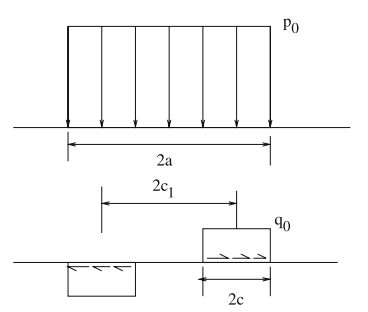

(a)

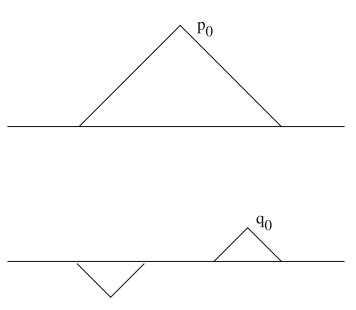

(b)

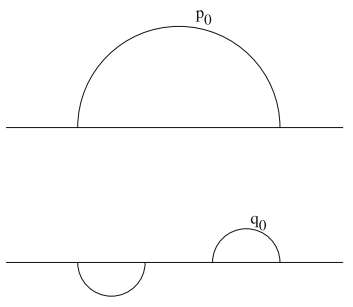

(c)

Figure 18. Fretting fatigue loading profiles.

Figure 17 (Ramesh et al 2007) shows the results for the case where a band of uniform pressure applied over $z / R, x / Y=-a$ to $a$ with $a=0.15 R$ and a shear band $\left(q_{0}=0.3 p_{0}\right)$ of width $0.1 R$ acts at a distance of $0.1 R$ from the origin (i.e. $c_{1}=0.1 R$ and $c=0.05 R$ ) as shown in figure 18. This particular combination simulates partial stick-slip interface which is commonly encountered in fretting (Dobromirsky \& Smith 1986). The stresses are shown normalised with applied pressure $\left|p_{0}\right|$ as mentioned in the figure labels. The stress values are matching well except for $\sigma_{\theta}$. Any difference in predicting $\sigma_{\theta}$ is probable to be reflected on $\tau_{o c t}$ which is important in determining the shear failure. $\tau_{o c t}$ at $r=0.99 R$ in the three different geometries are shown separately for pressure, shear and the combined fretting loading in figure 19. The figure shows that the prediction of $\tau_{o c t}$ at the near surface region in the cylinder by the 2D models is poor for the case of uniform pressure, good for the case of uniform shear and is moderate for the case of combined fretting loading. Similar results are shown for the cases of triangular and Hertzian profiles in figures 20 to 23 (Ramesh et al 2007).

The plots show that the models poorly predict $\tau_{o c t}$ only for uniform pressure. For the case of uniform shear and fretting loading the models predict $\tau_{o c t}$ well. The results shown here correspond to the case of $a / R=0 \cdot 15$. This ratio is expected to have considerable influence

(a)

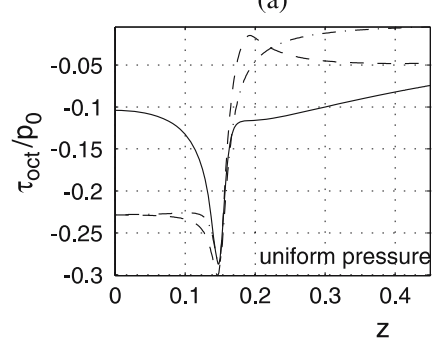

(c)

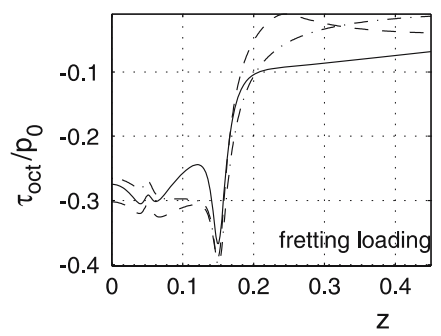

(b)

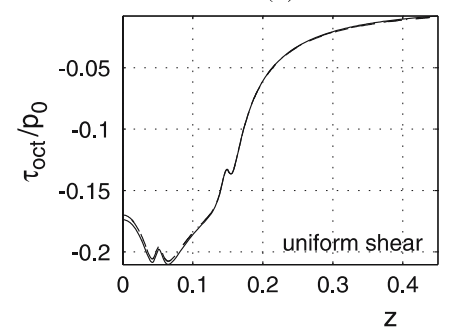

Figure 19. $\tau_{o c t}$ due to uniform pressure and shear. 
(a)
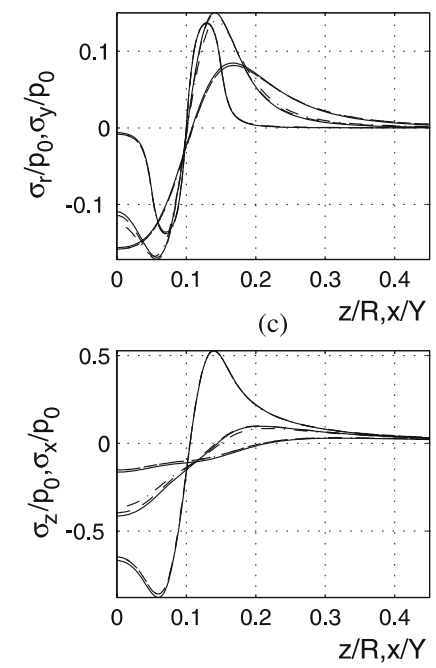

(b)

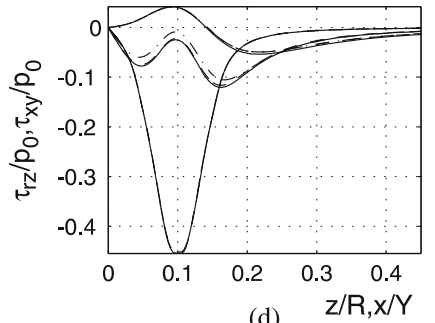

(d)

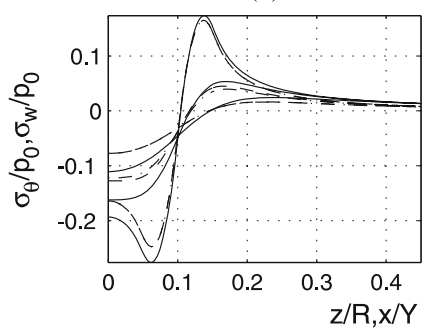

Figure 20. Stresses due to triangular pressure and shear.

on the stresses in near surface region and hence effect of $a / R$ needs to be studied in detail which is accomplished in the next paper (in this issue).

\section{Conclusion}

The results from the above study highlight the utility of evolving simplified strategies to understand the near surface states of stress. 2D Flamant analysis is obviously the simplest

(c)

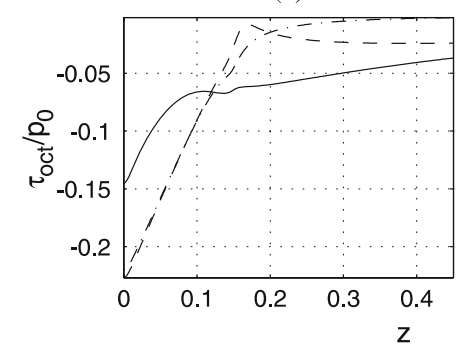

(c)

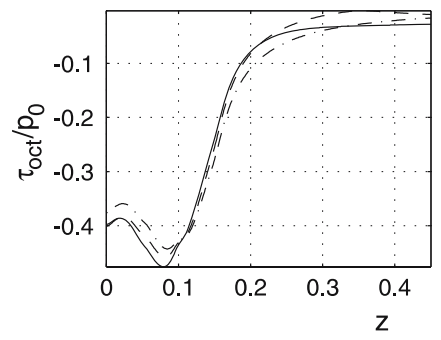

(c)

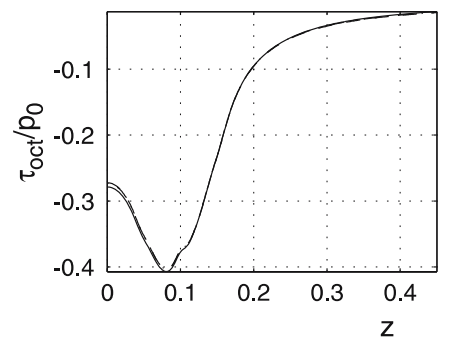

Figure 21. $\tau_{\text {oct }}$ due to triangular pressure and shear. 
(a)

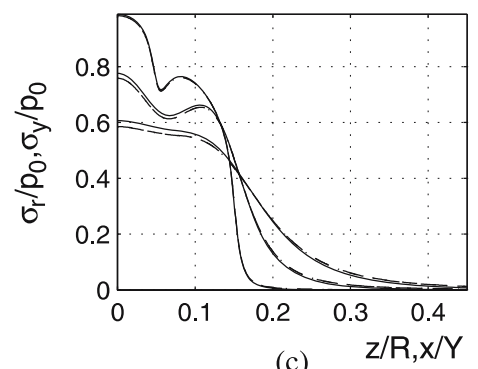

(c)

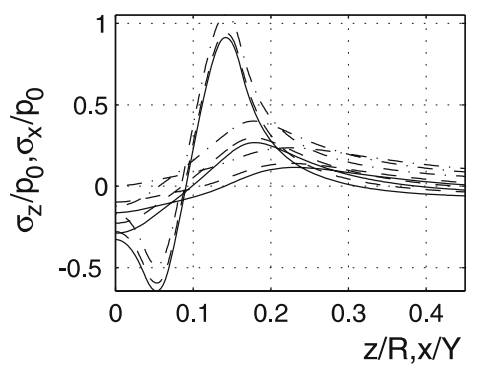

(b)

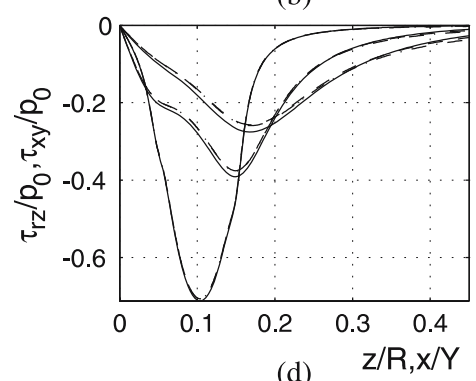

(d)

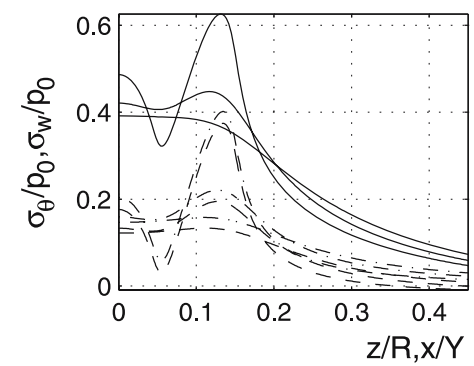

Figure 22. Stresses due to Hertzian pressure and shear.

strategy albeit inaccurate when it comes to predicting the axial stress $\sigma_{z}$. There is also a general disagreement between the out of plane stress predictions from 2DS and 2DF for assessing $\sigma_{\theta}$ in AFA. These discrepancies, however, disappear as the cylinder radius increases, and eventually coincide with AFA results for a cylinder of infinite radius. These simplified models are understandably not ideal, but could help designers in assessing fretting severity. After all, models never provide a complete and accurate picture of a complex process like fretting,

(a)

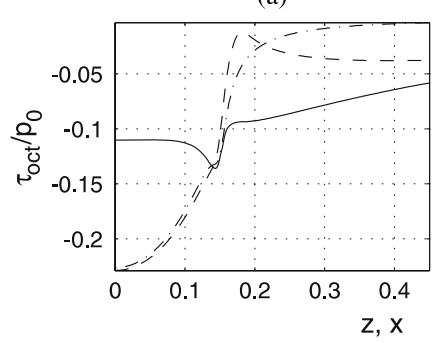

(c)

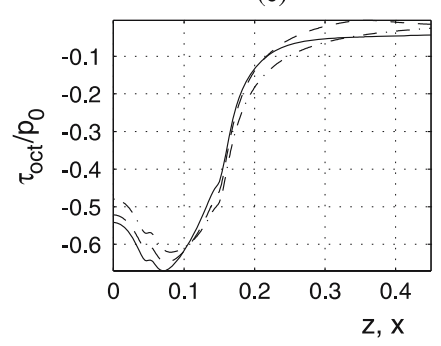

(b)

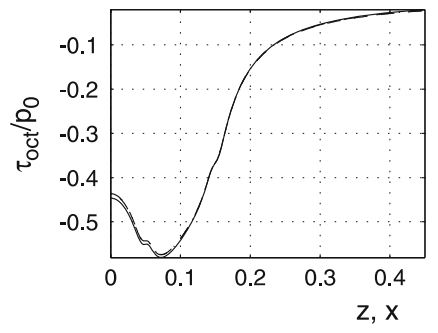

Figure 23. $\tau_{\text {oct }}$ due to Hertzian pressure and shear. 
but highlight critical aspects underlying mechanics and physics. Further work is necessary to extend the ideas presented here for thermal loading arising from fretting.

\section{References}

Bentall R H, Johnson K L 1967 Slip in the rolling contact of two dissimilar elastic rollers. Int. J. Mech. Sci. 9: 389-404

Boniface V, Simha K R Y 1991 A plastic zone model of mixed mode fracture. Int. J. Fract. 48: R9-R12

Conway H D, Farnham K A 1967 Contact stresses between cylindrical shafts and sleeves. Int. J. Eng. Sci. 5: 541-544

Dini D, Nowell D 2004 Flat and rounded fretting contact problems incorporating elastic layers. Int. J. Mech. Sci. 46: 1635-1657

Dobromirsky J, Smith I O 1986 A stress analysis of a shaft with a press-fitted hub subjected to cyclic axial loading. Int. J. Mech. Sci. 28(1): 41-52

Farris T N, Murthy H, Matlik J F 2003 Fretting fatigue, In R O Rithcie and Y Murakami, (eds.), Comprehensive structural integrity Elsevier, Pergamon Press 4: 281-326

Fellows L, Nowell D, Hills D A 1995 Contact stresses in a moderately thin strip (with particular reference to fretting experiments) Wear 185: 235-238

Hills D A, Nowell D Sackfield A 1993 Mechanics of elastic contacts (Oxford: Butterworth-Heinmann)

Johnson K L 1985 Contact mechanics. (Cambridge, UK: Cambridge University Press)

Lee D S 1995 Axisymmetric constriction of a circular cylinder under uniform axial compression. $Q$. J. Appl. Math. 48: 89-110

Lee H, Mall S 2004 Effect of dissimilar mating materials and contact force on fretting fatigue behaviour of Ti-6Al-4V. Tribol. Int. 37: 35-44

Mugadu A, Hills D A, Limmer L 2003 A theoretical and experimental procedure for predicting the fretting fatigue strength of complete contacts. Fretting fatigue: Advances in the basic understanding and applications ASTM-STP 1425: 145-155

Nowell D, Hills D A 1988 Contact problems incorporating elastic layers. Int. J. Solids and Struct. 24(1): 105-115

Ramesh M, Satish V Kailas, Simha K R Y 2007 Fretting stresses in axisymmetric components. National Conference of Research Scholars in Mechanical Engineering, Indian Institute of Technology Kanpur, India

Rankin A W, Schenectady N Y 1944 Shrink-fit stresses and deformations. J. Appl. Mech. A-77-A-85

Singh S 1994 On the shrink-fit problem of a thin cylinder. Int. J. Pressure Vessels and Piping 60: $167-175$

Sneddon I N 1951 Fourier Transforms. McGraw-Hill Book Company, Inc.

Spillers W R 1964 A shrink-fit problem. J. Math. Phys. 43: 65

Steven G P 1975 The shrink-fit problem with both components being elastic. Int. J. Eng. Sci. 13: 663-673

Szolwinski M P, Farris T N 1996 Mechanics of fretting fatigue crack formation. Wear 198: 93-107

Timoshenko S P, Goodier J N 1970 Theory of Elasticity. Engineering Mechanics Series. (New York: McGraw-Hill International Editions)

Tranter C J, Craggs J W 1945 The stress distribution in a long circular cylinder when a discontinuous pressure is applied to the curved surface. Philos. Mag. 36: 241-250

Williams D K 1996 Circular cylinders with bands of pressure: axisymmetric closed form solutions utilizing Bessel's functions and Fourier series. Pressure Vessels and Piping Design, Analysis and Severe Accidents 331: 77-87

Williams D K, Ranson W F 2003 Pipe-anchor discontinuity analysis utilizing power series solutions, Bessel's functions and Fourier series. Nucl. Eng. Des. 220: 1-10

Yau W W F, Cakmak A S 1966 The indentation problem of an infinite, hollow, elastic cylinder for an axisymmetric punch of finite length and arbitrary profile. Int. J. Eng. Sci. 4: 463-481 